\title{
Complex Visual Imagery and Cognition During Near-Death Experiences
}

\author{
Alexander Batthyány, PhD \\ University of Vienna \& International \\ Academy of Philosophy, Liechtenstein
}

\begin{abstract}
Near-death experiences (NDEs) entail complex and structured conscious experience during conditions known to coincide with rapid loss of consciousness often associated with decline or disruption of the neurological correlates currently held to be causative factors of visual imagery and cognition. In this study, $653 \mathrm{NDE}$ reports of cardiac and/or respiratory arrest patients were analyzed for unprompted, spontaneous references to quality of conscious visual imagery and mentation during an NDE. Results indicate that in a majority of NDEs, both figurative and abstract mentation are either preserved or markedly improved during unconsciousness and unresponsiveness in the context of respiratory and cardiac arrests. These findings underscore the call to further study the mechanisms behind the 'outliving' of a conscious sense of selfhood and complex, structured visual imagery and cognition during severely deteriorating physiological function-and perhaps especially during clinical death.
\end{abstract}

KEY WORDS: near-death experience, terminal lucidity, visual imagery; mentation; cognition; mind-body problem

Near-death experiences (NDEs) are phenomenologically complex experiences reported by $9-18 \%$ of patients who survived health crises

\footnotetext{
Alexander Batthyány, $\mathrm{PhD}$, is a professor who holds The Endowed Viktor Frankl Chair of Philosophy and Psychology at the International Academy of Philosophy in Bendern, Principality of Liechtenstein and who teaches theoretical foundations of cognitive science in the Department of Philosophy at the University of Vienna in Vienna, Austria. He would like to thank Barbora Gensorova, David Mandel, Petra Schmidt, and Johannes Berger for their assistance with handling the databases and scoring the reports. Special thanks go to Jeffrey Long of the Near-Death Experience Research Foundation (www.nderf.org) both for allowing use of NDERF experience repository data and for providing helpful remarks on earlier versions of this article. Warm thanks go also to Bruce Greyson for his kind support and his very helpful comments on an earlier draft and to Janice Holden for her support and editorial assistance. Correspondence regarding this article should be sent to Dr. Batthyány at Prinz Eugenstr. 18/12, 1040 Vienna, Austria, or email: alexander.batthyany@univie.ac.at.
} 
of varying severity (Greyson, 1998). Although researchers who conducted early field work (Moody, 1975) may have overestimated or overstated the case for a uniform structure and phenomenology of NDEs (Belanti, Perera, \& Jagadheesan, 2008; Kellehear, 1993; Knoblauch, Schmied, \& Schnettler, 2001), subsequent studies have confirmed several core features often, but not invariably, present during NDEs, such as the awareness of being dead, feelings of euphoria and peacefulness, a feeling of floating outside one's body, moving through a tunnel, and meeting deceased relatives or nonhuman entities (Athappilly, Greyson, \& Stevenson, 2006; Belanti et al., 2008). Whereas some of these features make for compelling narratives and are often evidential for experiencers (San Filipo, 2006), there is little consensus among researchers as to what triggers, causes, or underlies the experience and as to what the experience signifies (Agrillo, 2011; French, 2005).

Individual NDEs may differ in content and structure, but the one NDE-defining feature is the presence of conscious self-awareness and memory, and often complex imagery and cognition, during severe physiological crisis - often at a time during which patients are unresponsive and apparently unconscious. Yet only few researchers have looked into the reported quality of mentation during NDEs, and few have addressed the implications of the fact that near-death experiencers (NDErs) not only seem to retain a conscious sense of self and structured cognition but also often report enhanced, rather than diminished, alertness, imagery, and mentation during their NDEs (Greyson, 2010; Kelly, Greyson, \& Kelly, 2007). Patients may, for example, claim heightened mental clarity, vivid sensory imagery, and a clear memory of the experience during physiological crises frequently accompanied by hypoxia, hypercarbia, metabolic, and drug induced disturbances and seizures - that is, during states known to involve severely compromised cerebral function (Agrillo, 2011; Greyson, Kelly \& Kelly, 2009).

For instance, Emily Kelly, Bruce Greyson, and Edward Kelly (2007) conducted a questionnaire study with 800 NDErs asking multiple choice and narrative questions that specifically addressed changes in visual imagery and cognitive function during the experience. In their study, a full $80 \%$ of experiencers described their thinking during their NDEs as "clearer than usual" (45\%) or "as clear as usual" (35\%); furthermore, $65 \%$ recounted that their thinking during their NDEs was "as logical as usual" (36\%) or "more logical than usual" (29\%; Kelly, Greyson, \& Kelly, 2007, p. 386). Additionally, according to an earlier study, NDErs who reported complex mentation and imagery during their NDEs were significantly more often physiologically close to death than those who did 
not (Owens, Cook, \& Stevenson, 1990). In other words, in what appears to be at variance with what should be expected by current physicalist models of the mind, the more severe the physiological crisis, the more likely NDErs are to report having experienced clear and complex cognitive and sensory functioning.

The observation of intact, or even enhanced, visual imagery and mentation during presumed deterioration of structured neuronal functioning converges with two further death-related phenomena of seemingly intact or enhanced sensory and cognitive function in the presence of physiological crisis, namely terminal lucidity and "mindsight." Terminal lucidity refers to the unexpected return of mental clarity and memory shortly before death in patients with a wide variety of debilitating chronic mental and neurological disorders such as dementias, brain tumors and lesions, and chronic psychoses (Batthyány, 2014; Nahm \& Greyson, 2009; Nahm, Greyson, Kelly, \& Haraldsson, 2012). Mindsight refers to the return or the emergence of visual imagery during NDEs in subjects who are blind but who, during their NDEs, experience visual—and sometimes confirmed veridical-perceptions of physical objects and events (Ring, 2001; Ring \& Cooper, 1999). Next to anecdotes of veridical perception and knowledge acquisition during the out-of-body aspect of NDEs, these phenomena are often considered to support an "out-of-brain," that is, dualist interpretation of NDEs whereby mind and brain are considered to be ultimately independent entities (Facco \& Agrillo, 2012; Greyson, 2010; Grossmann, 2008).

However, the evidential quality of cases of terminal lucidity and mindsight currently hardly measures up to the scale of the philosophical implications on the nature and the functional relationship between brain and mind at death and dying. For one, both terminal lucidity and mindsight are rare occurrences; and secondly, even when they occur, researchers rarely get the opportunity to study them firsthand or at least in adequate temporal proximity and thus often have to rely on the accuracy (or not) of third party reports. As a testimony to how fragile this type of evidence can be, the first edition of Ring and Cooper's mindsight monograph (1999) had to carry a pasted-in disclaimer requesting readers to ignore one impressive case history of mindsight because additional probing raised the suspicion that this case report may have been fraudulent.

In brief, for the time being, the evidence for terminal lucidity and mindsight primarily refers to a few anecdotal and exceptional and occasionally ambivalent case reports scattered throughout the literature (Batthyány, 2014). Additionally, only a fraction of these reports have 
been written up with the research question in mind whether and under which circumstances complex sensory and mental function can coexist with concurrent loss, decline, or disintegration of brain function.

However, the results of Kelly at al. (2007) pose as much a challenge to physicalist models of the mind (and NDEs) as terminal lucidity and mindsight in that they, too, suggest that the conscious sense of selfhood and complex, structured visual imagery and mental clarity can "outlive" even severely altered brain function-including a flatline EEG. But in contrast to terminal lucidity and mindsight, the continuation of self and cognition despite often marked changes in supportive physiological parameters is the rule rather than the exception during NDEs.

At the same time, however, the notion of enhanced visual imagery, awareness, and mentation during NDEs currently is also based on a limited stock of data: Until now, only one unpublished study briefly reported in Kelly et al. (2007, p. 386) involved systematic investigation of the frequency of self-reports of enhanced meaningful and structured imagery, awareness, and mentation during NDEs.

In view of the implications of this phenomenon, we further explored self-reported cognitive and sensory function during NDEs in several large samples of first-person NDE accounts. Specifically, we investigated the reported state and potential changes of the quality of visual imagery and mentation during NDEs in large and independent samples of NDE reports in order to test the replicability of Kelly et al.'s finding. Additionally, in order to address the question whether complex visual imagery and mentation may be present during times of severe and life threatening states, only reports of subjects with medically diagnosed life threatening conditions with cardiac/respiratory arrest were included in this study. Our goal was to examine whether NDEs reported in the aftermath of these most severely life-threatening circumstances evidenced preserved or enhanced mental function.

\section{Method}

Our method consisted of simple text analysis of first person reports of NDEs. Reports were self-authored unsolicited volunteer narratives that came from four large repositories of NDE accounts. Three repositories were online: the database of the Near Death Experience Research Foundation (NDERF; www.nderf.org) with, at the time of our study, approximately 3,900 accounts of death- and crisis-related experiences; the International Association for Near-Death Studies 
(IANDS; www.iands.org) experience repository with approximately 290 accounts; and the Archive of Near-Death Experiences (www.near -death.com) with approximately 150 accounts. The fourth repository consisted of approximately 220 accounts of crisis experiences and possible NDEs from an earlier survey of ours (Saniga \& Batthyány, 2004). In total, we examined 4,572 reports-including what may have been a small number of duplicates or double-entries.

The reports were pooled and electronically scanned for explicit references to cognitive and visual function (e.g. saw, seen, vision, color, light, thought, thinking, idea, remember, memory). Reports that did not include these keywords were discarded. In a second step, the remaining subset of reports was scanned for references to the medical diagnosis and/or circumstances of the NDE (e.g. cardiac arrest, respiratory arrest, resuscitation, clinical death). The remaining reports with matching references were then individually read, double-checked for inclusion criteria, and, if they passed criteria, scored (see below).

Thus, to be included, an account had to meet two criteria: (a) It had to include at least one explicit reference to visual perception and/ or imagery and/or mentation, for example, "I saw the car wreck from about 3 meters above" and/or "I thought about my children"; and (b) it had to contain specific clinical information about the occurrence of the NDE such that the experiencer was unconscious and undergoing a diagnosed life-threatening cardiac or respiratory arrest. Out of the approximately 4,500 accounts that met the first criterion, only 2,319 carried specific medical information about the circumstances of the NDE, and, of those, 653 met the second criterion of a medically diagnosed life threatening cardiac/respiratory arrest. These 653 accounts were further analyzed as follows.

Given the exploratory design of the study and both the large number and diverse nature of the individual accounts, and in order to enable comparison with Kelly et al. (2007), we chose a simple and straightforward scoring method. All references to (a) visual perception or imagery and (b) alertness or mentation were rated on scales between -2 and +2 . Protocols were coded independently by three raters in a set of five reports at a time until at least $85 \%$ agreement was reached. The agreement criterion was reached after four sets. After that point, the remaining protocols were coded by individual raters. Disagreements or uncertain ratings were resolved through discussion. Pearson correlation revealed an inter-rater reliability of $r=.88$.

For both dimensions, i.e. visual imagery and alertness and/or mentation, only explicit mention of the respective dimension was scored. 
A score of 2 signified marked change, a score of 1 slight change, and a score 0 no reported change in the respective dimension; the operator ('+' or '-') designated qualitative increase or decrease of the respective dimension. For each report, each explicit reference relevant to one of the two dimensions was scored, and scores were then averaged so that for each dimension, the lowest possible average score was -2 (marked decline within the respective dimension) and the highest possible score +2 (marked enhancement in the respective dimension).

In brief, if a report included a reference to seeing and/or thinking (or synonyms thereof), it was scorable on the respective dimensions. If the report merely referred to seeing or thinking but did not include any additional descriptor or direct or indirect qualifier about the quality of seeing or thinking, seeming merely to describe seeing or thinking in a manner comparable to everyday wakefulness, it was scored as 0 ; for example, "I saw that my dad and step-mom had a worried look on their faces and tried to ask why but they did not hear me," and "I wondered whether this meant that I was dead and thought about my friends in the waiting area." Further examples of -2 (marked decline), -1 (slight decline), +1 (slight enhancement), and +2 (marked enhancement) appear in the Results section.

\section{Results}

Demographic data regarding age and gender were available for only $489(74.9 \%)$ of the 653 cases. Subjects in this subgroup were aged $18-74(M=53.7)$ and were $62.9 \%$ female and $37.1 \%$ male. In comparisons of females' and males' visual imagery scores as well as mentation scores, $t$ tests revealed no significant differences $(p>.05)$; thus, for analyses we did not differentiate by gender. Medical precursors of the NDE also varied widely and included, for example, clinical death not otherwise specified, surgery complications, organ failure, head injury, suicide attempts, anaphylactic shock, drowning or diving accidents, and car accidents. In 504 (77.2\%) of the total cases, the NDE-related health crisis had a sudden onset and was not preceded by chronic illness. In the remaining $22.8 \%$, subjects indicated that they had been physically unwell for a longer period of time and/or had expected an impending deterioration of health status before their NDE-related crises.

The input format for NDE reports differed considerably across the repositories, as some repositories included interview questions- whereas others merely asked subjects to describe their experience without fur- 
ther instructions. Accordingly, reports varied widely in length, precision, and density. Additionally, even within a repository, length and narrative structure of the reports varied considerably. Closer inspection within databases suggested that one potent factor on report length and descriptive depth was the length and descriptive precision of the immediate preceding reports of the respective entry - which were viewable to site visitors on two of the larger databases: NDERF and IANDS.

\section{Visual Imagery}

Out of the entire sample, only about 41 accounts (6.2\%) contained no explicit mention of any visual imagery but, instead, referred to inner affective states, mentation, and insights during the experience. These 41 accounts were therefore not scored on the visual imagery scale, leaving a reduced visual imagery sample of 612 accounts.

In this reduced sample $(n=612)$, only $73(11.9 \%)$ indicated that during at least one point in their NDE, their vision got considerably worse. Specifically, 18 subjects (2.9\%) reported temporary loss of vision, and 55 subjects $(8.9 \%)$ indicated markedly diminished vision. In the majority of these 73 cases, the experiencers attributed their diminished or loss of vision to their surroundings rather than to a loss or decline of vision per se; for example, "I had no vision; everything was dark"; "I could see nothing, it was complete darkness"; "It was very dark, and I only saw some shadows in a distance, but could not recognize who they were." Additionally, only eight subjects (1.3\% of the reduced sample; $10.9 \%$ of the diminished-vision group) reported loss, but not regain, of vision during their NDEs. The remaining 65 (10.6\% of the reduced sample; $89.1 \%$ of the diminished-vision sample) of those subjects who reported momentarily diminished or lost vision said that later in their NDEs, they experienced either a return of vision or an enhancement of visual imagery, such as when they experienced what appeared to them to be visually vivid scenery or encounters with deceased relatives, nonhuman persons, and/or lights. For example:

I was in the midst of utter warm darkness, nothing, only nothing. I was unafraid, but it was total darkness and warmth. After a while, I saw a tiny little ray of light piercing the darkness, then two, then dozens of them, and soon found myself bathing in multi-colored light rays. It was the most extraordinary thing I ever saw. Not only 'seeing' it, actually, but living in it.

Visual imagery rating of those 65 subjects who reported a return of vision after a temporary loss or decline showed that $42(64.6 \%)$ indi- 
cated slightly enhanced vision (a score of +1), and 23 (35.4\%) indicated marked improvement of vision (+2), with none recounting a return of normal, vision.

The majority of subjects in the entire reduced sample who explicitly mentioned visual imagery — 539 (88\% of the visual imagery sample) made no reference to such a temporary loss or decline of visual ability. Of these, 252 (41.1\% of the scored sample) were scored 0 as they did not explicitly mention of change in visual quality but merely reported visual experiences without any additional qualifier. The remaining 287 subjects $(46.9 \%)$ reported either a mild (149 subjects; $24.4 \%)$ or a marked (139 subjects; 22.5\%) improvement of their vision during their NDEs. For example, these narratives scored +2: "No need for glasses anymore! I saw perfectly well without them," and

It was entirely different than 'seeing' as you would define it. 'Seeing' there is in entirety. The images were created in my knowing through great vibrations, all energy expressing itself through different frequencies. Light was used to send thoughts into me, which then formed images, but not before my eyes, in my mind as though I had seen it.

\section{Cognition}

Fewer subjects spontaneously mentioned state and quality of cognition than reported visual imagery. Out of the entire sample of 653 experiences, about a third (226 accounts; $34.6 \%$ ) contained no explicit mention of cognitive processes during the experience. A majority of these reports (191 cases; 84.5\%), however, contained explicit or implied reference to often complex content at least suggestive of normal cognitive functioning. For example: "It was so beautiful but I also longed to be with my children. Leaving them alone at this early age seemed overwhelmingly painful." The following analyses refer to the reduced sample of the remaining 427 reports that contained explicit references to alertness and mentation, as only these could be scored according to the scoring scheme.

Only one single subject $(0.23 \%)$ indicated a marked decrease of alertness and mentation:

I just felt as if half asleep. Whenever 'I woke up' during the time I was out of my body, I found the outlook of survival simply too tiring and not very exciting at all and fell back into what I would compare to a fitful half-sleep.

Two subjects (0.46\%) reported a slight decline in mentation, but not in alertness: "Very present and awake, but also extremely relaxed. 
My thinking was strangely viscous and slow. [. . . I simply wanted to watch this beautiful warm yellowish light";

I felt peaceful, receptive. Was totally empty, but in a very positive and elated way. 'Finally I am at home'-I repeated over and over again like a mantra. Nothing much happened inside me-all happened out there and I watched and was happy.

Eleven subjects $(25.8 \%)$ indicated that they were in a trance- or dream-like state of consciousness with strong affective connotations but with what appeared to be slight declines in alertness or mentation: "The journey felt like a dream, but it wasn't a dream and the feelings were extremely intense, but dreamy"; "it reminded me of my student days when we took acid: mind-blowing." These types of responses were scored - 1 to indicate a slight negative change in alertness and/ or cognition.

A total of 262 subjects ( $61.4 \%$ of the reduced scorable sample) mentioned being conscious of their self, their environments, and mentation but did not use any further qualifying adjectives or any indication of either decline or enhancement of the scored dimension; these were scored 0 . The remaining 151 subjects (35.4\% of the scorable group) reported slight $(70 ; 16.4 \%)$ or marked $(81 ; 19 \%)$ heightening of awareness and alertness and clearer thinking: "I felt extremely aware, totally present, sharp and focused. In hindsight, it's like being half asleep when I was alive, and totally awake after I was pronounced dead";

My mind felt cleared and my thoughts seemed quick and decisive. I felt a great sense of freedom and was quite content to be rid of my body. I felt a connection with everything around me in a way that I cannot describe. I felt as if I was thinking faster or that time had slowed down considerably.

Whereas the three subjects who reported a decline on the alertness and mentation dimension indicated change either in alertness or mentation only, but not in both (see above), we found no clear trend of such a separation of both concepts in either the "marked enhancement" or in the "slight enhancement" groups.

\section{Discussion}

In this text analysis of a large sample of NDE narratives, we found that a substantial number of subjects spontaneously reported slightly or markedly enhanced visual imagery during their NDEs, and only a minority remembered loss or decline of vision, though in the majority 
of these latter cases this reduction was attributed to the surroundings in which the NDErs found themselves rather than to the subjects' own visual abilities per se. Additionally, the majority of those subjects who reported a momentary decline in visual imagery said that as their NDEs unfolded in time, vision returned and frequently entailed complex and detailed imagery suggestive of unchanged or enhanced vision - and did not revert to diminished levels. Secondly, we found that an overwhelming majority of subjects recounted either no change in, or slightly or markedly enhanced alertness and mentation during their NDEs, and only a minority remembered a decline in mentation or alertness. These results did not vary by gender. Although the nature of the data precluded further statistical analysis, it was our informal observation that these results did not vary by other demographic or by circumstantial variables. It remains for future researchers to confirm or disconfirm our informal observation through formal analysis.

\section{Comparison With Earlier Studies}

The results of this study confirm and replicate Kelly et al.'s (2007) report on quality of thinking during NDEs with a large independent sample of NDErs who underwent a severe medical crisis; they additionally suggest that complex visual imagery and cognition are highly prevalent in the typical NDE. Together, these results indicate that in a majority of NDEs, both figurative and abstract mentation is either preserved or improved during unconsciousness and unresponsiveness in the context of likely respiratory and cardiac arrest.

Before we enter a discussion of how our results compare to results of earlier similar studies, it should be noted that our methodologyrating of spontaneous comments on the quality of vision and cognition in brief self-reports - was expected to yield very conservative estimates of the scored dimensions in comparison to results generated by explicitly asking subjects to comment on the quality of the scored dimensions as Kelly et al. (2007) did. Additionally, our "no change" ratings differed considerably from Kelly et al.'s (2007). Given that we scored spontaneous reports, we had to account for the relatively large group of subjects who explicitly mentioned visual imagery and/ or thinking but who did not use any qualifying adjectives suggesting either decline or enhancement. As these reports included no mention of change on the mentation dimension, we scored these neutral reports as 0, i.e., no explicit reference to change. But as Greyson pointed out, 
"failing to use further adjectives is not the same as reporting alertness or mentation as normal; it is just a failure to specify whether cognition was normal, enhanced or diminished" (B. Greyson, personal communication, September 20, 2015). It is therefore very likely that our scoring method inflated the frequency of "no change" ratings. In contrast, Kelly et al.'s subjects received a no-change rating if they explicitly stated that functionality of the scored dimension was "as usual," that is, not much different from everyday waking life. Hence our and Kelly et al.'s "no change" groups are not directly comparable.

In Kelly et al.'s (2007) sample, 52.2\% reported that their vision "got clearer than usual," $32.2 \%$ described their vision as "as clear as usual," and 5.6\% as "not as clear as usual" (B. Greyson, personal communication, September 20, 2015). In our sample of unprompted reports of change in visual imagery during the NDE, $46.9 \%$ described a slight or marked enhancement of visual imagery, $41.1 \%$ mentioned visual imagery without any further qualifier - thus receiving a score of 0 , and $11.9 \%$ indicated a temporary decline or loss of vision-which in most cases experiencers attributed to external circumstances rather than to a decline of visual ability per se. Hence despite differences in scoring methods, the results of our study roughly replicate Kelly et al.'s results on this dimension.

Secondly, Kelly et al. (2007) reported that $45 \%$ of their subjects indicated that their thinking was clearer than usual, and $35 \%$ of experiencers described their thinking "as clear as usual"; thus $80 \%$ of their subjects indicated either no change or enhancement in cognition. Additionally, Jeffrey Long (J. Long, personal communication, September 16,2015$)$ found that in a group of 1,122 contributors to the NDERF repository, when directly asked, "How did your highest level of consciousness and alertness during the experience compare to your normal every day consciousness and alertness?", 74.4\% reported "more consciousness and alertness," 20.4\% reported unchanged consciousness and alertness during their NDEs, and only 5.2\% described their consciousness and alertness as less than normal

As to be expected with our scoring method, we found a substantially higher rate of unrated or presumably unchanged (61.4\%) mentation, but we also found a surprisingly large number of spontaneous reports of enhanced (35.4\%) alertness and mentation during the NDE, whereas only $3.3 \%$ reported a downturn in alertness and mentation. Although results of both studies are somewhat similar, our data pointed to a higher incidence of no change in cognition during 
NDEs - with a difference of 26 percentage points - which is not surprising given the difference between the studies in operationalization of "no change."

At the same time, our results are even more striking if one takes into account that reports of changes in the scored dimensions were mostly spontaneous and unprompted. Whereas a number of researchers have implicated expectancy effects to influence the narratives of NDE accounts (Belanti, Perera, \& Jagadheesan, 2008; Kellehear, 1993; Knoblauch, Schmied, \& Schnettler, 2001), it is unlikely that such effects unduly influenced or biased the results reported here. Enhanced visual ability, alertness, and mentation do not seem to be central elements of the classical NDE narrative. Additionally, as pointed out in the results section, a number of accounts clearly indicated that subjects were engaging in often highly complex integrative cognitive processing during their NDEs and yet did not explicitly comment on changes in alertness or mentation. Others did not explicitly indicate change in visual processing, and yet their accounts included often highly complex and vivid visual imagery-such as otherworldly landscapes or colors never seen before. Though in both cases it is reasonable to infer that visual imagery and alertness or mentation were either unchanged or enhanced during the NDEs, we nonetheless refrained from scoring such accounts on the respective dimensions and, thus again, risked under- rather than overestimation of changes in the two scored dimensions. The trade-off between caution and false-negatives was not the only limitation of this study, however.

\section{Limitations}

Like every retrospective survey study, this study, too, has a number of shortcomings and limitations, which we shall address next. First of all, the accounts used in this study were not elicited in order to study the dependent variables investigated in the foregoing analyses-which again is more likely to have led to under- rather than to over-reporting of changes in these dimensions-that is, Type II rather than Type I error. Our results are therefore likely to provide an overly conservative estimate of the developments in the dimensions in our sample.

Secondly, respondents were self-selected volunteers and thus may not have been representative of NDErs as a whole. Although we cannot rule out that our results were somehow biased by the self-selection of subjects, a growing number of researchers have found that online surveys and repositories generate data as reliable and valid, and some- 
times even more so, than data generated by more traditional interview methods (Lindhjem \& Navrud, 2011; Riva, Teruzzi \& Anolli, 2003; Weigold, Weigold \& Russell, 2013).

Still, we cannot rule out that one or another of the accounts included in our sample might have been fraudulent or fake; however, on NDERF, the largest contributor of NDEs studied here, less than 1\% of posted NDEs have been removed due to concerns about their validity (J. Long, personal communication, September 27, 2015; see also Long \& Long, 2003). Additionally, given the sheer number of accounts, it is unlikely that fake reports have significantly biased our results in one or the other direction. One would expect fake accounts either to be nonsensical-in which case the report wouldn't have met inclusion criteria-or, if the fake was an intentional fraud, to be prototypical of the popular NDE narrative. As pointed out above, however, enhanced visual imagery, alertness, and mentation do not seem to be central elements of the popular NDE narrative; hence, one might reasonably infer that fake reports would not have over-proportionally indicated change in the dimensions investigated in this study. Thus, whereas it is possible that some fake reports might have passed our inclusion criteria, they are unlikely to have significantly affected our main results.

A third potential weakness of our study concerns inclusion criteria, such as the medical conditions related to the NDEs. We tried to apply strict inclusion criteria, such as clear indication of respiratory and/or cardiac arrest, and therefore discarded a large number of reports that, although they included detailed medical information suggestive of lifethreatening conditions, did not elaborate sufficiently on cardiac and respiratory status. We thus excluded an indefinite number of accounts that, under more lenient inclusion criteria, would have deserved to be analyzed. One potential criticism is that perhaps our inclusion criteria may have skewed results towards particularly detailed reports in general, and subjects who gave more detailed and longer reports about their medical status during their NDEs might have therefore also over-reported (in comparison to a more representative sample) visual imagery and mentation.

To address this concern, we conducted informal test scorings of 40 randomly selected accounts that included some medical informationbut not sufficient for inclusion-which were suggestive of a severe health crisis during the NDEs, but found no indication that results would have been different had we included less detailed accounts. In fact, given that cardiac arrests are often followed by post-arrest confusion and amnesia (Aminoff, Scheinman, Griffin, \& Herre, 1988), it is 
again likely that our strict inclusion criteria skewed results towards under- rather than over-reporting of positive changes in mentation and visual imagery during the NDEs in our sample.

Fourth, it must be conceded that our scoring dimensions were fairly crude. For example, throughout this study, we consistently were confronted with the question of whether and how we were to distinguish between visual ability (i.e. 'seeing') and visual imagery (i.e. the 'seen'); in the end we had to recognize that with retrospective reports of the kind studied here, any distinction of this kind would have been far too ambiguous and uncertain to add exploratory value to this study. In comparison to, for example, the more impressive and perhaps also evidential mindsight cases-veridical perception during the NDEs of people with physical blindness - our visual imagery findings are therefore certainly less compelling. But they are considerably more frequent, and it should be remembered that the purpose of our analysis was not to investigate the veracity of visual perceptions during the NDE but, rather, to examine the question whether complex and structured-that is, meaningful and coherent-visual imagery can occur in the presence of severe physiological crisis and, presumably, cerebral dysfunction. For this question, we found strong support.

The same objective guided the choice of our second dimension: alertness and mentation. Clearly, both elements of this dimension are related, yet evidently they are distinguishable-the former referring to a state, the latter to functions and processes. Accordingly, at the beginning of this study we originally planned to distinguish between the two elements and score them separately. However, it turned out that here, too, we could not easily uphold this distinction without convoluting analysis at the cost of precision. Very few accounts allowed for a clear distinction between self-reported changes in alertness on the one hand and mentation on the other hand-with the exception of the tiny fraction of reports that indicated a marked decrease of alertness and/ or mentation.

Within the framework of this study, however, it was the unchanged, slight, or marked increase in alertness and mentation during cardiac and/or respiratory arrest-related NDEs that was of interest to us; again, we found strong support for the notion of normal to enhanced alertness and thought in a substantial number of NDErs pre-selected on the basis of the severity of their health condition during their NDEs, thus replicating earlier results with a large sample of over 650 individual case reports.

Last but not least, it should also be noted that retrospective stud- 
ies such as this one leave open the possibility that the NDEs-and with them, the continuity or enhancement of the scored dimensionsoccurred during the peak of their health crises, that is, during cardiac or respiratory arrest or perhaps shortly thereafter when presumably cerebral function was more structured and stable and thus supportive of clear(er) consciousness, thinking, and sense of selfhood. We cannot rule out that an undetermined number of the experiences (or parts of some of the experiences) analyzed in this study may have taken place in close proximity, but not, or not only, precisely during the physiological insult. However, shortly after cardiac or respiratory arrest, brain function and cognitive function are known to be severely compromised, and subjects are typically confused and amnesic about events immediately preceding and following the peak of the health crisis (Aminoff et al., 1988). Thus, even if an indefinite number of reports may have referred to visual and cognitive function briefly after, rather than during, the crisis, the restriction of including only cases of cardiac or respiratory failure is likely to have worked against rather than for finding such a high proportion of unprompted reports of continued or enhanced visual imagery and mentation during the NDEs—or memories thereof after the NDEs.

Moreover, one may compare our findings to awareness during anesthesia-especially considering that the majority of subjects in this study were in emergency treatment during their health crisis and hence likely to have received anesthetics and/or sedatives. However, the stark differences between cognitive status in NDEs and during anesthesia suggest that lucidity during NDEs and awareness in anesthesia or similar states are two very different phenomena. For instance, in contrast to awareness during or immediately after cardiac and respiratory arrest, awareness during general anesthesia is extremely rare- $9-18 \%$ vs. $0.1-0.5 \%$, respectively, and, phenomenologically, in contrast to the normal or enhanced cognitive function reported by a substantial proportion of our subjects, memory following anesthesia usually consists of a vague auditory recall or a sense of dreaming (Myles, Leslie, McNeil, Forbes, \& Chan, 2004). Additionally, memories of awareness during general anesthesia may take several days to weeks to become consciously accessible (Nordstrom, Engstrom, Persson, \& Sandin 1997), whereas NDEs are usually recalled immediately upon return of full functional consciousness, and memories of the experience are remarkably stable and consistent even years after the experience (Greyson, 2007; van Lommel, van Wees, Meyers, \& Elfferich, 2001). The available evidence thus indicates that 
there is something rather unique about consciousness during NDEs in comparison to consciousness in related conditions.

\section{Conclusions}

In sum, despite of the presence of a number of methodological factors that, in all likelihood, skewed results towards underestimation of no or positive changes in the scored dimensions, we found in this study a strikingly large number of mostly unprompted, spontaneous reports of continuation or slight or marked enhancement in visual imagery and mentation during the NDEs of a group of subjects who would otherwise have been expected to suffer from loss or deterioration in cognitive, sensory, and memory function during as well as in the aftermath of a cardiac and/or respiratory arrest. The results of this study therefore indicate that the conscious sense of selfhood and complex, structured visual imagery and mental clarity can, under certain circumstances, "outlive" even severely altered brain function-including a flatline EEG or post-arrest trauma. Hence, converging with the related phenomena of terminal lucidity and mindsight, these data indicate the possibility of the continuation of self and mental function despite often marked decline in supportive physiological parameters. In contrast to the comparatively rare incidents of mindsight and terminal lucidity, however, our results suggest that the continuity of visual imagery, mentation, and sense of selfhood is the rule rather than the exception during NDEs.

Together with case studies of terminal lucidity and mindsight, our findings of enhanced mentation and visual imagery during severe physiological crises appear to therefore indicate that, at least near death, the relationship between cognition, perception, and their neuronal correlates might be more complex than traditionally thought. Specifically, the findings reported here and in earlier studies do not square well with physicalist reductionist models of the mind but, prima facie, appear to support the alternative dualist proposition that at least during extraordinary intervening circumstances such as the dying process, mind and brain may emerge as two different entities with separable, or indeed separate, functions (Elitzur, 2009). As briefly pointed out in the introduction, the basic argument for a dualist interpretation is relatively straightforward: According to physicalist theories of the mind, conscious awareness, selfhood, and mentation are products of, or identical with, their respective neuronal correlates (Crick \& Koch, 1990; Place, 1956). Within this framework, NDEs, too, 
are held to be caused by neurophysiological processes at or around the dying process (Augustine, 2007), such as cerebral anoxia (Lempert, Bauer, \& Schmidt, 1994), cerebral hypoxia (Blackmore \& Troscianko, 1988), cerebral hypercarbia, releases of endorphins (Carr, 1981), or glutamate release accompanied by blockades of NMDA receptors (Jansen, 1989). The findings reported in this paper and cases of terminal lucidity and mindsight, however, appear to suggest otherwise in that they indicate the presence of complex and structured conscious experience during decline, breakdown, or absence of the neurobiological correlates commonly held to be causative factors of NDEs-and of conscious experience in general.

As Greyson (2010) pointed out,

the most important objection to the adequacy of all reductionist psychophysiological theories . . is mental clarity, vivid sensory imagery, a clear memory of the experience, and a conviction that the experience seemed more real than ordinary consciousness are the norm for NDEs, even when they occur in conditions of drastically altered cerebral physiology under which the reductionist model would deem consciousness impossible (p. 39).

The results of this study therefore underscore the call to further study the mechanisms behind the seeming continuation of a conscious sense of selfhood and complex, structured visual imagery and mental clarity during altered brain function and severely deteriorating physiological activity in general-and perhaps especially during clinical death. Clearly, further systematic investigation of this phenomenon is warranted, especially given its implications for the question of the authenticity of NDEs and the relationship of mind and brain at death.

\section{References}

Agrillo, C. (2011). Near-death experience: Out-of-body and out-of-brain? Review of General Psychology, 15(1), 1-10.

Aminoff, M. J., Scheinman, M. M., Griffin, J. C., \& Herre, J. M. (1988). Electrocerebral accompaniments of syncope associated with malignant ventricular arrhythmias. Annals of Internal Medicine, 108, 791-796.

Athappilly, G. K., Greyson, B., \& Stevenson, I. (2006). Do prevailing societal models influence reports of near-death experiences? A comparison of accounts reported before and after 1975. Journal of Nervous and Mental Disease, 94, $218-222$.

Augustine K. (2007). Psychophysiological and cultural correlates undermining a survivalist interpretation of near-death experiences. Journal of Near-Death Studies, 26, 89-125.

Batthyány, A. (2014). The Light before the end of the tunnel. Preliminary data 
from the European study on terminal lucidity. Presentation at the Annual Conference of the International Association for Near-Death Studies, Newport Beach, CA.

Belanti, J., Perera, M., \& Jagadheesan, K. (2008). Phenomenology of neardeath experiences: A cross-cultural perspective. Transcultural Psychiatry, 45, 121-133.

Blackmore, S. J., \& Troscianko, T. (1988). The physiology of the tunnel. Journal of Near-Death Studies, 8, 15-28.

Carr, D. B., \& Prendergast, M. (1981). Endorphins at the approach of death. The Lancet, 317, 390. doi:10.1016/S0140-6736(81)91714-1

Crick, F., \& Koch, C. (1990) Towards a neurobiological theory of consciousness. Seminars in the Neurosciences, 2, 263-275.

Elitzur, A. C. (2009). Consciousness makes a difference: A reluctant dualist's confession. In A. Batthyány \& A. C. Elitzur (Eds.), Irreducibly conscious. Selected papers on consciousness. Heidelberg, Germany: Universitätsverlag Winter.

Facco, E., \& Agrillo, C. (2012). Near-death experiences between science and prejudice. Frontiers in Human Neuroscience. 6(209), 1-7.

French, C. C. (2005). Near-death experiences in cardiac arrest survivors. Progress in Brain Research, 150, 351-367.

Greyson, B. (1998). The incidence of near-death experiences. Medicine and Psychiatry, 1, 92-99.

Greyson, B. (2007). Consistency of near-death experience accounts over two decades: Are reports embellished over time? Resuscitation, 73, 407-411.

Greyson, B. (2010). Implications of near-death experiences for a postmaterialist psychology. Psychology of Religion and Spirituality, 2, 37-45.

Greyson, B., Kelly, E. W., \& Kelly, E. F. (2009). Explanatory models for neardeath experiences. In J. Holden, B. Greyson, \& D. James (Eds.), The handbook of near-death experiences (pp. 213-234). Santa Barbara, CA: Praeger/ ABC-CLIO.

Grossmann, N. (2008). Four errors commonly made by professional debunkers. Journal of Near-Death Studies, 26, 231-238.

Jansen, K. L. R. (1989). Near-death experience and the NMDA receptor. British Medical Journal, 298, 1708-1709.

Kellehear, A. (1993). Culture, biology, and the near-death experience: A reappraisal. Journal of Nervous and Mental Disease, 181, 148-156.

Kelly, E. W., Greyson, B., \& Kelly, E. F. (2007). Unusual experiences near death and related phenomena. In E. F. Kelly, E. W. Kelly, A. Crabtree, A. Gauld, M. Grosso, \& B. Greyson, Irreducible mind (pp. 367-421). Lanham, MD: Rowman \& Littlefield.

Knoblauch, H., Schmied, I., \& Schnettler, B. (2001). Different kinds of neardeath experience: A report on a survey of near-death experiences in Germany. Journal of Near-Death Studies, 20, 15-29.

Lempert, T., Bauer, M., \& Schmidt, D. (1994). Syncope and near-death experience. The Lancet, 344, 829-830.

Lindhjem, H., \& Navrud, S. (2011). Are Internet surveys an alternative to face-to-face interviews in contingent valuation? Ecological Economics, 70(9), $1628-1637$.

Long, J., \& Long, J. (2003). A comparison of NDEs occurring before and after 
1975. Results from a Web survey of near death experiencers. Journal for NearDeath Studies, 22, 21-32.

Mobbs, D., \& Watt, C. (2011). There is nothing paranormal about near-death experiences. Trends in Cognitive Science. 15, 447-449.

Myles, P. S., Leslie, K., McNeil, J., Forbes, A., \& Chan, M. T. (2004). Bispectral index monitoring to prevent awareness during anaesthesia: The B-Aware randomized controlled trial. The Lancet, 363, 1757-1763.

Moody, R. A. (1975). Life after life. Covington, GA: Mockingbird Books.

Nahm, N., \& Greyson, B. (2009). Terminal lucidity in mental disorders. Journal of Nervous and Mental Disease, 197, 942-944.

Nahm, M., Greyson, B., Kelly, E. W., \& Haraldsson, E. (2012). Terminal lucidity: A review and case collection. Archives of Gerontology and Geriatrics, 55, $138-142$.

Nordstrom O., Engstrom A., Persson S., \& Sandin R. (1997). Incidence of awareness in total i.v. anaesthesia based on propofol, alfentanil and neuromuscular blockade. Acta Anaesthesiologica Scandinavia, 41, 978-84.

Owens, J. E., Cook, E. W., \& Stevenson, I. (1990). Features of "near-death experience" in relation to whether or not patients were near death. The Lancet, 336, 1175-1177.

Place, U. T. (1956). Is consciousness a brain process? British Journal of Psychology, 47, 44-50.

Ranta, S. O., Herranen, P., \& Hyxynen, M. (2002). Patient's conscious recollections from cardiac anaesthesia. Journal of Cardiothoracic and Vascular Anesthesia, 16, 426-30.

Ring, K. (2001). Mindsight: Eyeless vision in the blind. In D. Lorimer (Ed.), Thinking beyond the brain: A wider science of consciousness (pp. 59-70). Edinburgh, Scotland, UK: Floris Books.

Ring, K., \& Cooper, S. (1999). Mindsight: near-death and out-of-body experiences in the blind. Palo Alto, CA: William James Center for Consciousness Studies.

Riva, G., Teruzzi, T., \& Anolli, T. (2003). The use of the Internet in psychological research: Comparison of online and offline questionnaires. CyberPsychology and Behavior, 6(1), 73-80.

San Filippo, D. (2006). Religious interpretations of death, afterlife and near-death experiences. Faculty Publications. Paper 32. Chicago, IL: National Louis University. Retrieved from http://digitalcommons.nl.edu/faculty_publications/32/

Saniga, M., \& Batthyány, A. (2005). I didn't want to return. Extraordinary time perception and physical time. Unpublished manuscript.

Sebel, P. S., Bowdle, T. A., Ghoneim, M. M., Rampil, I. J., Padilla, R. E., Gan, T. J., \& Domino, K. B. (2004). The incidence of awareness during anaesthesia: A multicenter United States study. Anesthesia \& Analgesia, 99, 833-839.

van Lommel, P., van Wees, R., Meyers, V., \& Elfferich, I. (2001). Near-death experience in survivors of cardiac arrest: A prospective study in the Netherlands. The Lancet, 358, 2039-2045.

Weigold, A., Weigold, I. K., \& Russell, E. J. (2013). Examination of the equivalence of self-report survey-based paper-and-pencil and Internet data collection methods. Psychological Methods, 18(1), 53-70. 